\title{
Patología quirúrgica emergente durante la pandemia COVID-19 en un hospital de segundo nivel en Ecuador
}

\author{
Emergency surgical disease during the COVID-19 pandemic in a second level hospital \\ in Ecuador
}

\section{Patologia cirúrgica emergente durante a pandemia de COVID-19 em um hospital de segundo nível no Equador}

\author{
Sebastián Alejandro Pástor Romero \\ sebastian.pastor.md@gmail.com \\ ORCID: 0000-0001-9401-0389
}

Juan Carlos Cárdenas Dávalos

juan_carlos_c_88@hotmail.com

ORCID: 0000-0002-8916-8576

\author{
Paola Alexandra Medina Flores \\ paola.medina.md@gmail.com \\ ORCID: 0000-0001-5796-4714
}

\author{
Walter Barba Bermeo \\ walterb038@icloud.com \\ ORCID: 0000-0001-8087-0054
}

Hospital General Riobamba, Instituto Ecuatoriano de Seguridad Social, Ecuador

Recibido 02 de septiembre 2020 | Arbitrado y aceptado 24 de septiembre 2020 | Publicado 22 de diciembre 2020

\begin{abstract}
RESUMEN
Introducción: Desde la declaración de pandemia por la OMS el once de marzo de 2020, ha existido un descenso en los ingresos de pacientes quirúrgicos por la paralización de la cirugía electiva y la disminución de procedimientos quirúrgicos emergentes. Objetivo: Analizar la incidencia y características de la patología quirúrgica emergente en un hospital de segundo nivel en Ecuador. Material y métodos: Estudio retrospectivo, descriptivo, que incluyó a todos los pacientes sometidos a cirugía general emergente en el Hospital General Riobamba IESS, desde el once de marzo hasta el once de agosto de 2020. Resultados: En el estudio se incluyeron 63 pacientes, con una media de edad de 48,54 años; el 50,8\% fueron hombres. El diagnóstico más frecuente fue apendicitis aguda en $61,9 \%$ casos. Se realizaron 66 cirugías, $36,5 \%$ fueron apendicectomías convencionales. El abordaje abierto se llevó a cabo en $68,2 \%$ de los pacientes. El $12,7 \%$ de pacientes presentaron COVID-19 en el perioperatorio de los cuales $25 \%$ fallecieron. La mortalidad general fue del $7,9 \%$ y todos los pacientes fallecidos fueron hombres. Discusión: La evidencia acerca del manejo quirúrgico durante la pandemia COVID-19 aún es limitada. La patología quirúrgica emergente no se puede diferir, se deben tener en cuenta el incremento en la mortalidad de pacientes infectados y el riesgo de exposición del equipo quirúrgico. Conclusión: Durante la pandemia se produjo un descenso en las cirugías realizadas en nuestro centro, la apendicitis aguda continúa siendo la principal patología quirúrgica emergente. La mortalidad se asoció al sexo masculino y diagnóstico de COVID-19.
\end{abstract}

Palabras clave: COVID-19; cirugía general; laparoscopía; urgencias médicas

\begin{abstract}
Introduction: Since the declaration of a pandemic by the WHO on March 11, 2020, there has been a decrease in the admissions of surgical patients due to the paralyzation of elective surgery and the decrease in emerging surgical procedures. Objective: To analyze the incidence and characteristics of emerging surgical pathology in a second level hospital in Ecuador. Material and methods: Retrospective and descriptive study, which included all the patients undergoing emergency general surgery at Hospital General Riobamba IESS, from March 11 to August 11, 2020. Results: 63 patients were included in the study, with a mean age of 48.54 years; $50.8 \%$ were men. The most frequent diagnosis was acute appendicitis in $61.9 \%$ cases. 66 surgeries were performed, $36.5 \%$ were conventional appendicectomies. The open approach was carried out in $68.2 \%$ of the patients. $12.7 \%$ of patients presented COVID-19 in the perioperative period, of which $25 \%$ died. Overall mortality was $7.9 \%$ and all deceased patients were men. Discussion: The evidence about surgical management during the COVID-19 pandemic is still limited. Emerging surgical pathology cannot be deferred, the increased mortality of infected patients and the risk of exposure of the surgical team must be taken into account. Conclusion: During the pandemic there was a decrease in the surgeries performed in our center, acute appendicitis continues to be the main emerging surgical pathology. Mortality was associated with male sex and diagnosis of COVID-19.
\end{abstract}

SP: Médico General por la Universidad Nacional de Chimborazo (2011), Especialista en Cirugía General por la Universidad Central del Ecuador (2018) Magister en Docencia Universitaria por la Universidad de las Fuerzas Armadas ESPE (2017), Cirujano General del Hospital General Riobamba IESS, profesor temporal de la cátedra de Cirugía Práctica en la Universidad Nacional de Chimborazo Instituto Ecuatoriano de Seguridad Social Hospital General Riobamba. Ecuador.

PM: Médica General por la Universidad Nacional de Chimborazo (2011). Especialista en Medicina de Emergencias y Desastres por la Universidad Central del Ecuador (2018). Estudiante de la maestrí en Gerencia de Instituciones de salud en la Universidad Técnica Particular de Loja Médico tratante del servicio de Emergencia del Hospital General Riobamba IESS Instituto Ecuatoriano de Seguridad Social Hospital General Riobamba. Ecuador.

JC: Médico Cirujano por Universidad Tecnológica Equinoccial (2014), Médico residente del servicio de Cirugía General de Hospital General Riobamba IESS desde el año 2016. Instituto Ecuatoriano de Seguridad Social - Hospital General Riobamba. Ecuador.

WB: Médico General por la Escuela Superior Politécnica de Chimborazo (2014). Médico Residente del servicio de Cirugía General del Hospital General Riobamba IESS Instituto Ecuatoriano de Seguridad Social Hospital General Riobamba. Ecuador.

Key words: COVID-19; general surgery; laparoscopy; medical emergencies 
SP: Médico General por la Universidad Nacional de Chimborazo (2011) Especialista en Cirugía General por la Universidad Central del Ecuador (2018), Magister en Docencia Universitaria por Universidad de las Fuerzas Armadas ESPE (2017) Cirujano General del Hospital General Riobamba IESS, profesor temporal de la cátedra de Cirugía Práctica en la Universidad Nacional de Chimborazo Instituto Ecuatoriano de Seguridad Social Hospital General Riobamba. Ecuador.

PM: Médica General por la Universidad Nacional de Chimborazo (2011) Especialista en Medicina de Emergencias y Desastres por la Universidad Central del Ecuador (2018). Estudiante de la maestrí en Gerencia de Instituciones de salud en la Universidad Técnica Particular de Loja. Médico tratante del servicio de Emergencia del Hospital General Riobamba IESS. Instituto Ecuatoriano de Seguridad Social Hospital General Riobamba. Ecuador.

IC: Médico Cirujano por Universidad Tecnológica Equinoccial (2014), Médico residente del servicio de Cirugía General del Hospital General Riobamba IESS desde el año 2016. Instituto Ecuatoriano de Seguridad Social - Hospital General Riobamba.

WB: Médico General por la Escuela Superior Politécnica de Chimborazo (2014). Médico Residente del servicio de Cirugía General del Hospital General Riobamba IESS Instituto Ecuatoriano de Seguridad Socia Hospital General Riobamba. Ecuador.

\section{RESUMO}

Introdução: Desde a declaração de uma pandemia pela OMS em 11 de março de 2020, houve uma diminuição nas admissões de pacientes cirúrgicos devido à paralisação das cirurgias eletivas e diminuição dos procedimentos cirúrgicos emergentes. Objetivo: Analisar a incidência e as características da patologia cirúrgica emergente em um hospital de segundo nível no Equador. Material e métodos: Estudo retrospectivo, descritivo, que incluiu todos os pacientes submetidos à cirurgia geral de urgência no Hospital Geral de Riobamba IESS, no período de 11 de março a 11 de agosto de 2020. Resultados: O estudo incluiu 63 pacientes, com média de idade de 48,54 anos; $50,8 \%$ eram homens. O diagnóstico mais frequente foi apendicite aguda em $61,9 \%$ dos casos. Foram realizadas 66 cirurgias, 36,5\% foram apendicectomias convencionais. A abordagem aberta foi realizada em $68,2 \%$ dos pacientes. $12,7 \%$ dos pacientes apresentaram COVID-19 no período perioperatório, dos quais $25 \%$ morreram. A mortalidade geral foi de $7,9 \%$ e todos os pacientes falecidos eram homens. Discussão: As evidências sobre o manejo cirúrgico durante a pandemia de COVID-19 ainda são limitadas. A patologia cirúrgica emergente não pode ser adiada, o aumento da mortalidade dos pacientes infectados e o risco de exposição da equipe cirúrgica devem ser levados em consideração. Conclusão: Durante a pandemia houve diminuição das cirurgias realizadas em nosso centro, a apendicite aguda continua sendo a principal patologia cirúrgica emergente. A mortalidade foi associada ao sexo masculino e ao diagnóstico de COVID-19.

Palavras-chave: COVID-19; cirurgia geral; laparoscopia; emergência médica

\section{INTRODUCCIÓN}

$\mathrm{E}$ n diciembre de 2019 en Wuhan, provincia de Hubei, China, se reportó por primera vez una enfermedad respiratoria causada por el nuevo coronavirus que posteriormente fue denominado como SARS-CoV-2, y la enfermedad catalogada como COVID-19, que al momento de la realización de este artículo ha afectado a más de 30 millones de personas a nivel mundial. (1) En Ecuador, según los datos publicados por el Ministerio de Salud Pública, hasta el 19 de septiembre del 2020 se han reportado 125.620 casos confirmados por RT-PCR y 11.084 muertes. (2)

Desde la aparición del primer caso de COVID-19 en nuestro país y la declaración de pandemia por la OMS el once de marzo de 2020 (3), ha existido un descenso en los ingresos de pacientes con patología quirúrgica, principalmente por la paralización de la consulta externa y la cirugía electiva (4), aunque también ha existido un descenso de los procedimientos quirúrgicos emergentes, a pesar de que estos no se pueden diferir. Además, cabe mencionar que ha sido evidente la priorización de la atención de pacientes infectados con COVID-19, lo que ha llevado a la adaptación de la infraestructura física de los sistemas de salud y hospitales para afrontar la demanda de estos pacientes. En el Hospital General Riobamba se adecuaron algunos quirófanos para funcionar como Unidades de Cuidados Intensivos provisionales, a esto se le puede sumar la redistribución del personal de salud, tanto médicos como enfermeras, para cubrir la atención de pacientes infectados con el nuevo coronavirus. 
Las medidas que se han tomado para disminuir los procedimientos quirúrgicos tienen el objetivo de minimizar la exposición del personal de salud al virus (5) y reducir el riesgo de infección nosocomial por COVID-19 en los pacientes quirúrgicos.

Es importante tomar en cuenta que a pesar de que hasta el momento se han publicado varios estudios sobre la incidencia de patología quirúrgica durante la pandemia, la evidencia aún es limitada en este campo, y no existen datos locales. La mayoría de datos provienen de estudios de cohorte y casos controles, lo que nos lleva a la necesidad de investigar el impacto que ha tenido la pandemia en la atención de pacientes quirúrgicos emergentes en nuestra población.

El objetivo principal de este estudio es analizar la incidencia y características de la patología quirúrgica emergente en un hospital de segundo nivel en el Ecuador, lo que nos permitirá tener una visión más clara de la situación local, y que podría ser de utilidad para la elaboración de protocolos perioperatorios para el manejo de pacientes quirúrgicos con y sin COVID19 asociado, que garanticen la seguridad del paciente y del personal de salud.

\section{MATERIALES Y MÉTODOS}

$\mathrm{S}$ e realizó un estudio retrospectivo, descriptivo, que incluyó a todos los pacientes que fueron sometidos a cirugía general emergente en el Hospital General Riobamba IESS, desde el once de marzo, fecha en que la OMS declaró al COVID-19 como pandemia, hasta el once de agosto de 2020. Se analizaron los datos demográficos, diagnóstico pre $\mathrm{y}$ postquirúrgico, el tipo de intervención quirúrgica, tipo de anestesia administrada, días de estancia hospitalaria postquirúrgica y la presencia o no infección por SARS-CoV-2 en el momento de la cirugía, catalogada como tal dependiendo de los datos clínicos, RT-PCR o serología positiva y/o tomografía simple de tórax sugestiva para COVID-19. Los datos se obtuvieron tras consultar los informes digitalizados en el sistema AS400 del Instituto Ecuatoriano de Seguridad Social. Además, se revisó la incidencia de la patología quirúrgica emergente en el mismo periodo de tiempo durante el año 2019 con el fin de comparar el número de cirugías realizadas.

\section{RESULTADOS}

$\mathrm{E}$ $n$ el estudio se incluyeron un total de 63 pacientes, con una media de edad de 48,54 +/- 18,4 años, de los cuales $32(50,8 \%)$ fueron hombres y 31 $(49,2 \%)$ mujeres. El diagnóstico más frecuente fue apendicitis aguda en 39 $(61,9 \%)$ casos, seguido de $6 \quad(9,5 \%)$ pacientes con colecistitis aguda, $4(6,3 \%)$ pacientes con vólvulo de sigma entre otros diagnósticos que están resumidos en la tabla 1. 
Tabla 1. Distribución demográfica y principales diagnósticos

\begin{tabular}{lcc}
\hline Variables & $\begin{array}{c}\text { Frecuencia (número) } \\
\text { Media, DE }\end{array}$ & Porcentaje (\%) \\
\hline Sexo & 32 & 50,8 \\
$\quad$ Hombres & 31 & 49,2 \\
$\quad$ Mujeres & $48,54+/-18,4$ & \\
Edad (a) & 5 & 7,9 \\
Mortalidad & & \\
Diagnóstico & 39 & 61,9 \\
Apendicitis aguda & 6 & 9,5 \\
Colecistitis aguda & 4 & 6,3 \\
Vólvulo de sigma & 2 & 3,2 \\
$\quad$ Hernia inguinal incarcerada & 2 & 3,2 \\
Hernia ventral incarcerada & 2 & 3,2 \\
Hernia interna & 1 & 1,6 \\
Absceso perianal & 1 & 1,6 \\
Cuerpo extraño en recto & 1 & 1,6 \\
Fístula traqueoesofágica iatrogénica & 1 & 1,6 \\
Sepsis de foco abdominal por dehiscencia & & \\
del muñón intestinal & 1 & 1,6 \\
Sindrome pilorico & 1 & 1,6 \\
Síndrome adherencial por EPI & 1 & 1,6 \\
Trauma abdominal & 1 & 1,6 \\
Tumor hemorrágico de cola de páncreas & & \\
* El paciente fue intervenido de manera emergente por complicación de hemicolectomía \\
derecha electiva por tumor colónico.
\end{tabular}

Se realizaron un total de 66 cirugías debido a dos relaparotomías en una paciente que presentó un tumor hemorrágico de cola de páncreas y una reintervención en un paciente con fístula traqueoesofágica iatrogénica para gastrostomía de alimentación. Se realizaron 23 $(36,5 \%)$ apendicectomías convencionales, 15 (23,8\%) apendicectomías laparoscópicas, 6 $(9,5 \%)$ colecistectomías laparoscópicas entre otras múltiples intervenciones en dependencia de la patología de base, las mismas que se hallan resumidas en la tabla 2. 
Tabla 2. Características de los procedimientos quirúrgicos realizados

\begin{tabular}{ccc}
\hline Variables & $\begin{array}{c}\text { Frecuencia } \\
\text { (número) }\end{array}$ & $\begin{array}{c}\text { Porcentaje } \\
\text { (\%) }\end{array}$ \\
\hline
\end{tabular}

Tipo de anestesia

General

Raquídea

Duración de la anestesia

Días postquirúrgicos

Diagnóstico

\section{2}

24

$120(80-150)$

$1,98(1,26-3,25)$

Apendicectomía convencional

Apendicectomía laparoscópica

Colecistectomía laparoscópica

Hernioplastia convencional

Sigmoidectomía + Hartmann

LE + apendicectomía

$\mathrm{LE}+$ adhesiolisis

$\mathrm{LE}+$ hernia ventral

$\mathrm{LE}+$ pexia de sigma

LE + rafia de colon

$\mathrm{LE}+$ rafia intestinal

LE + resección intestinal + ileostomía

Herniorrafia ventral

Adhesiolisis +yeyunostomía de alimentación

Drenaje de absceso perianal

Extracción de cuerpo extraño rectal

Exploración cervical + traqueostomía / Gastrostomía de alimentación *

LE + esplenectomía / Relaparotomía + rafia intestinal /

Relaparotomía + lavado + drenaje $†$

*Paciente reintervenido por fístula traqueoesofágica y posteriormente se realizó gastrostomía de alimentación

†Paciente intervenida por tumor sangrante de cola de páncreas, que posteriormente presentó fístula duodenal y fue reintervenida por dos ocasiones 
La mayoría de las intervenciones se realizaron bajo anestesia general, en 42 $(63,6 \%)$ pacientes, mientras que 24 $(36,4 \%)$ de los procedimientos fueron realizados con anestesia raquídea. La mediana de la duración de la anestesia fue de 120 (P25-P75 = 80-150) minutos. El abordaje laparoscópico se llevó a cabo en 21 $(31,8 \%)$ pacientes, mientras que $45(68,2 \%)$ pacientes fueron sometidos a cirugía abierta. La estancia mediana de hospitalización postquirúrgica fue de 1,98 (P25-P75 = 1,26 3,25) días.

Un total de $8 \quad(12,7 \%)$ pacientes presentaron COVID-19 en el perioperatorio, de los cuales fallecieron 2 (25\%) pacientes, mientras que la mortalidad general fue del $7,9 \%$, destacando que todos los pacientes fallecidos fueron de sexo masculino. La mortalidad se correlacionó con diagnóstico de COVID-19 y el sexo con un coeficiente de Pearson $(p<0,05)$ de 0,007 y 0,037 respectivamente.

\section{DISCUSIÓN}

$\mathrm{L}$ a evidencia acerca del manejo quirúrgico durante la pandemia COVID-19 aún es escasa, y es indiscutible que ha constituido un reto muy grande para los sistemas de salud, hospitales, servicios quirúrgicos $\mathrm{y}$ profesionales que trabajan con pacientes que cursan con patologías emergentes. En muchos centros asistenciales ha sido necesario enfocar los recursos institucionales en la atención de pacientes afectados por esta enfermedad, lo que ha supuesto el cierre de áreas quirúrgicas, suspensión de cirugía electiva e incluso apoyo de cirujanos y personal quirúrgico en áreas COVID-19.

La patología quirúrgica emergente no se puede diferir a pesar de la pandemia, pero se deben tener en cuenta los riesgos de complicaciones perioperatorias y el riesgo de exposición que implica para el equipo quirúrgico. Los resultados del presente estudio demuestran una disminución evidente de la actividad quirúrgica emergente en comparación al mismo periodo de tiempo en el 2019, donde se realizaron 261 intervenciones, lo que corresponde a una reducción del $74,7 \%$ de procedimientos quirúrgicos. Pérez-Rubio et al. (6) reportaron una disminución del $14 \%$ de patología abdominal urgente potencialmente quirúrgica, aunque existen reportes internacionales donde se ha observado disminución del 52,7\% comparado con la producción del mismo mes en relación al año anterior (7) y hasta el $86 \%$ en cirugías emergentes durante el primer mes de pandemia comparado con datos del mes anterior al inicio de la cuarentena $(8,9)$. Esto puede ser producto de que muchos pacientes evitan las casas de salud que atienden pacientes con COVID-19 por temor a infectarse, los casos leves no acudieron al hospital probablemente padeciendo su proceso nosológico en el domicilio y en varios casos se refirieron pacientes a otras instituciones de salud para la resolución de su cuadro.

Existió una ligera predominancia del sexo masculino, similar a lo reportado por Cano-Valderrama et al, además al igual que en este estudio y otros reportes internacionales el diagnóstico más frecuente fue apendicitis aguda $(9,10)$, lo que no ha cambiado a pesar de la pandemia. La mediana de la duración de la anestesia fue mayor a lo reportado en otros estudios (9), probablemente debido al mayor tiempo que implica para el equipo quirúrgico cumplir con el protocolo 
establecido en la institución y el uso correcto de los equipos de protección personal.

No existe evidencia científica de transmisión de SARS-Cov-2 por vía laparoscópica (11,12), en el hospital donde se llevó a cabo este estudio se realizaron $31,8 \%$ de procedimientos por esta vía, un porcentaje menor al 57,82\% descrito en reportes internacionales (10), por lo que continúa siendo una buena opción por sus múltiples ventajas para el paciente, incluido el menor tiempo de hospitalización que podría influir en disminuir el riesgo de infecciones nosocomiales y la exposición del personal de salud. (5) Se observó una estancia de hospitalización postquirúrgica corta, cercana a los dos días, menor a lo reportado en otros estudios (10), posiblemente en relación con lo ya mencionado anteriormente.

Según la corte internacional CovidSurg (13) la mortalidad rodea el $25,6 \%$ en cirugía de emergencia y se menciona al sexo masculino como predictor de mortalidad a 30 días, lo cual está en relación con lo encontrado en el presente estudio en el que además llama la atención que todos los fallecidos fueron hombres.

Esta investigación reveló algunas limitantes, ya que al realizarse en un hospital de segundo nivel el universo es relativamente pequeño, además se trató de un estudio con diseño retrospectivo lo que pudo ser una debilidad, algunos pacientes con patologías potencialmente quirúrgicas recibieron tratamiento conservador y no fueron incluidos, así mismo, la pandemia sigue en curso y pueden existir cambios influenciados por el comportamiento social, los decretos estatales $y$ administrativos.

\section{CONCLUSIONES}

$\mathrm{D}$ urante la pandemia se ha producido un descenso en el número de cirugías realizadas en el Hospital General Riobamba por varios motivos; la apendicitis aguda continúa siendo la principal patología quirúrgica emergente; la laparoscopía es una buena opción para el manejo quirúrgico durante la pandemia; el sexo masculino y el diagnóstico de COVID-19 se asociaron con mayor mortalidad. Es necesario analizar las implicaciones en morbimortalidad a mediano y largo plazo en futuros trabajos, además de la aparente relación con la mayor mortalidad en hombres y el impacto en el pronóstico de los pacientes infectados con SARS-Cov-2 sometidos a cirugías de urgencia.

- Conflicto de intereses. Ninguno declarado por los autores.

- Financiación. Los costos fueron asumidos por los autores sin financiación externa de ningún tipo.

- Agradecimiento. Al Hospital General Riobamba del Instituto Ecuatoriano de Seguridad Social.

- Investigación realizada considerando los tratados bioéticos

\section{REFERENCIAS BIBLIOGRÁFICAS}

1. Dong E, Du H, Gardner L. An interactive web-based dashboard to track COVID19 in real time. Lancet Inf Dis [Internet]. 2020 Feb [citado 2020 Sept 18] 20(5):533-534. Disponible en: https://doi.org/10.1016/S14733099(20)30120-1

2. Ecuador. Situación Nacional por COVID19 [Internet]. Ministerio de Salud Pública. Infografía $\mathrm{N}^{\circ} 205 ; 2020$ [citado 2020 Sept 19]. Disponible en: https://www.salud.gob.ec/wp- 
content/uploads/2020/09/INFOGRAFI A-NACIONALCOVID19-COE-NACIONAL08h00-19092020.pdf

3. Organización Mundial de la Salud. Cronología de la respuesta de la OMS a la COVID-19 [Internet]. OMS; 2020 [citado 2020 Sept 18]. Disponible en: https://www.who.int/es/newsroom/detail/29-06-2020-covidtimeline

4. Spinelli A, Pellino G. COVID-19 pandemic: perspectives on an unfolding crisis. Br J Surg [Internet]. 2020 Mar [citado 2020 Sept 18] 107: 785-787. Disponible en: https://doi.org/10.1002/bjs.11627

5. De Simone B, Chouillard E, Di Saverio S, Pagani L, Sartelli M, Biffl WL, et al. Emergency surgery during the COVID19 pandemic: what you need to know for practice. Ann R Coll Surg Engl [Internet]. 2020 May [citado 2020 Sept 19] 102(5):323-332. Disponible en: https://doi.org/10.1308/rcsann.2020.0 097

6. Pérez-Rubio Á, Sebastián Tomás JC, Navarro-Martínez S, Gonzálvez Guardiola P, Torrecillas Meroño DG, Domingo Del Pozo C. Incidence of surgical abdominal emergencies during SARS-CoV-2 pandemic. Cir Esp [Internet]. 2020 Jul [citado 2020 Sept 19] 7:S0009-739X(20)30224-4. Disponible en: https://doi.org/10.1016/j.ciresp.2020.0 6.017

7. Álvarez Gallego $M$, Gortázar de Las Casas S, Pascual Migueláñez I, RubioPérez I, Barragán Serrano C, Álvarez Peña E, el al. SARS-CoV-2 pandemic on the activity and professionals of a General Surgery and Digestive Surgery Service in a tertiary hospital. Cir Esp [Internet]. 2020 Jun-Jul [citado 2020 Sept 19] 98(6):320-327. Disponible en: https://doi.org/10.1016/j.ciresp.2020.0 4.001

8. Patriti A, Eugeni E, Guerra F. What happened to surgical emergencies in the era of COVID-19 outbreak?
Considerations of surgeons working in an Italian COVID-19 red zone. Updates Surg [Internet]. 2020 Jun [citado 2020 Sept 19] 72(2):309-310. Disponible en: https://doi.org/10.1007/s13304-02000779-6

9. Tagleb S, Mazahreh A, Abdelwahab J, Aleshawi, Nabil A, et al. The impact of COVID-19 on the surgical operations. Ann Med Surg [Internet]. 2020 Sep [citado 2020 Sept 18] 57: 49-51. Disponible en: https://doi.org/10.1016/j.amsu.2020.06 .042

10. Cano-Valderrama $O$, Morales $X$, Ferrigni CJ, Martín-Antona E, Turrado V, García A, el al. Acute Care Surgery during the COVID-19 pandemic in Spain: Changes in volume, causes and complications. A multicentre retrospective cohort study. Int J Surg [Internet]. 2020 Aug [citado 2020 Sept 19] 80:157-161. Disponible en:

https://doi.org/10.1016/j.ijsu.2020.07.0 02

11. El Boghdady $M$, Ewalds-Kvist $B$. Laparoscopic Surgery and the debate on its safety during COVID-19 pandemic: A systematic review of recommendations. T Surg [Internet]. 2020 Jul [citado 2020 Sept 19] Disponible en: https://doi.org/10.1016/j.surge.2020.07 .005

12. Tivey D, Davis $S$, Kovoor J, Babidge $W$, Tan L, Hugh T, el al. Safe surgery during the coronavirus disease 2019 crisis. ANZ J Surg [Internet]. 2020 Jun [citado 2020 Sept 19] 90: 1553-1557. Disponible en: https://doi.org/10.1111/ans.1608

13. CovidSurg Collaborative. Mortality and pulmonary complications in patients undergoing surgery with perioperative SARS-CoV-2 infection: an international cohort study. Lancet [Internet]. 2020; 4;396(10243):27-38 [citado 2020 Sept 19] Disponible en: https://doi.org/10.1016/S01406736(20)31182-X 